\title{
Estimation of electronic coupling in $\pi$-stacked donor-bridge-acceptor systems: Correction of the two-state model
}

\author{
Alexander A. Voityuk ${ }^{\mathrm{a})}$ \\ Institució Catalana de Recerca i Estudis Avançats, Passeig Lluis Companys 23, Barcelona 08010, Spain \\ and Institute of Computational Chemistry, Universitat de Girona, 17071 Girona, Spain
}

(Received 3 October 2005; accepted 20 December 2005; published online 13 February 2006)

\begin{abstract}
Comparison of donor-acceptor electronic couplings calculated within two-state and three-state models suggests that the two-state treatment can provide unreliable estimates of $V_{\mathrm{da}}$ because of neglecting the multistate effects. We show that in most cases accurate values of the electronic coupling in a $\pi$ stack, where donor and acceptor are separated by a bridging unit, can be obtained as $\widetilde{V}_{\mathrm{da}}=\left(E_{2}-E_{1}\right) \mu_{12} / R_{\mathrm{da}}+\left(2 E_{3}-E_{1}-E_{2}\right) 2 \mu_{13} \mu_{23} / R_{\mathrm{da}}^{2}$, where $E_{1}, E_{2}$, and $E_{3}$ are adiabatic energies of the ground, charge-transfer, and bridge states, respectively, $\mu_{i j}$ is the transition dipole moments between the states $i$ and $j$, and $R_{\mathrm{da}}$ is the distance between the planes of donor and acceptor. In this expression based on the generalized Mulliken-Hush approach, the first term corresponds to the coupling derived within a two-state model, whereas the second term is the superexchange correction accounting for the bridge effect. The formula is extended to bridges consisting of several subunits. The influence of the donor-acceptor energy mismatch on the excess charge distribution, adiabatic dipole and transition moments, and electronic couplings is examined. A diagnostic is developed to determine whether the two-state approach can be applied. Based on numerical results, we showed that the superexchange correction considerably improves estimates of the donor-acceptor coupling derived within a two-state approach. In most cases when the two-state scheme fails, the formula gives reliable results which are in good agreement (within 5\%) with the data of the three-state generalized Mulliken-Hush model. (C) 2006 American Institute of Physics.

[DOI: $10.1063 / 1.2166232$ ]
\end{abstract}

\section{INTRODUCTION}

Bridge-mediated electron transfer (ET) in $\pi$ stacks has recently attracted much attention because of its importance in several physical and biological processes. For instance, organic solar cells which are fabricated by sandwiching an organic layer between two electrodes are the subject of intensive experimental and theoretical investigations. ${ }^{1}$ Another example is a long-range charge migration in DNA. ${ }^{2}$ The efficiency of ET between donor and acceptor sites (or electrodes) can be essentially regulated by variation of the molecular structure of the bridge. Theoretical and computational studies provide very useful results which allow one to elucidate the role of electronic, structural, and dynamic factors in ET through $\pi$ stacks. ${ }^{3,4}$ The effective coupling of donor and acceptor $V_{\mathrm{da}}$ is a key parameter which determines the efficiency of the ET reactions, and therefore quantummechanical calculations of this parameter is of wide interest. $^{5,6}$ The application of different computational schemes to estimate $V_{\mathrm{da}}$ in DNA stacks has been discussed recently. $^{7}$

Several years ago, Cave and Newton introduced the generalized Mulliken-Hush (GMH) method. ${ }^{8,9}$ This scheme employs a transformation of adiabatic states to diabatic states that diagonalizes the dipole moment matrix. The GMH method has been employed for estimating electronic couplings in various systems. In most studies, a two-state ap-

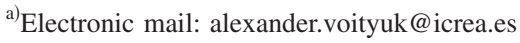

proach was used. Within this model, the electronic coupling can be expressed via the vertical excitation energy $E_{2}-E_{1}$, the transition dipole moment $\mu_{12}$, and the difference of diabatic dipole moments of donor and acceptor $\mu_{a}-\mu_{d}$,

$$
V_{\mathrm{da}}=\left(E_{2}-E_{1}\right) \frac{\mu_{12}}{\mu_{a}-\mu_{d}}=\left(E_{2}-E_{1}\right) \frac{\mu_{12}}{\sqrt{\left(\mu_{1}-\mu_{2}\right)^{2}+4 \mu_{12}^{2}}} .
$$

In turn, the difference $\mu_{a}-\mu_{d}$ can be estimated by using only adiabatic dipole moment matrix elements, $\mu_{a}-\mu_{d}$ $=\sqrt{\left(\mu_{1}-\mu_{2}\right)^{2}+4 \mu_{12}^{2}}$. The two-state GMH method allows calculation of electronic couplings in various systems, independent of symmetry and geometrical constraints. ${ }^{8,9}$ This approach has been successfully applied to biologically relevant $\pi$ stacks. Beljonne et al. employed the scheme to study photoinduced hole transfer in DNA hairpins. ${ }^{10}$ Zheng et al. calculated electronic couplings in $\pi$-stacked porphyrinbridge-quinone systems. ${ }^{11}$ Very recently, the GMH scheme has been applied to derive electronic couplings for excess electron transfer in DNA. ${ }^{12}$

An important advantage of the GMH method is that it is able to deal with systems where more than two adiabatic states enter into the description of relevant diabatic states. ${ }^{8,9}$ When the diabatic states of interest are represented by a combination of more than two adiabatic states, a multistate model should be employed. So far, only several papers which deal with the multistate GMH approach have been published. Rust et al. considered in detail the case with three states, 
where two diabatic states (the ground state and a locally excited state) are localized on the donor and a single-electron transfer state is localized on the acceptor. ${ }^{13}$ They developed a diagnostic for determining when a third state has to be considered within GMH calculations. Recently, a multistate GMH approach has been employed for calculating electronic couplings for charge transfer in DNA $\pi$ stacks consisting of three, four, and five base pairs. ${ }^{14}$ It was found that although for some systems the two-state scheme provides reasonable estimates, in general, this model fails to reproduce the electronic couplings calculated with the multistate approach. ${ }^{14}$ Using a three-state GMH scheme, Lambert et al. derived electronic couplings from spectroscopic data and semiempirical calculations for some organic radical cations. ${ }^{15}$ They analyzed the dependence of $V_{\mathrm{da}}$ on adiabatic parameters and suggested that the two-state model is a good approximation only if the difference of dipole moments of donor and acceptor is large compared to transition moments associated with a bridge state. However, we will see that a diagnostic based only on comparison of adiabatic dipole moments can be misleading.

In this paper, we suggest a simple formula which takes into account the effects of the bridge state, develop a diagnostic to determine when a multistate treatment must be employed, and compare donor-acceptor electronic couplings calculated using the introduced formula with the corresponding values derived from the two- and multistate GMH models.

\section{RESULTS AND DISCUSSION}

\section{Relationship between electronic couplings derived within the two- and three-state frameworks}

Let us consider a donor-bridge-acceptor system, where two adiabatic states of the lowest energy, $\psi_{1}$ and $\psi_{2}$ correspond to donor and acceptor and an adiabatic state $\psi_{3}$ is essentially localized on the bridge; $E_{1}, E_{2}$, and $E_{3}$ are energies of these states. In the adiabatic representation, the Hamiltonian matrix is diagonal,

$$
\left(\begin{array}{ccc}
E_{1} & 0 & 0 \\
0 & E_{2} & 0 \\
0 & 0 & E_{3}
\end{array}\right) .
$$

Within the three-state model, the adiabatic states $\psi_{1}, \psi_{2}$, and $\psi_{3}$ are transformed into diabatic states $\bar{\varphi}_{d}, \bar{\varphi}_{a}$, and $\bar{\varphi}_{b}$ of donor, acceptor, and bridge, respectively. The unitary transformation of the adiabatic Hamiltonian results in a symmetric matrix

$$
\left(\begin{array}{ccc}
\bar{\varepsilon}_{d} & \bar{V}_{\mathrm{da}} & \bar{V}_{\mathrm{db}} \\
\bar{V}_{\mathrm{da}} & \bar{\varepsilon}_{a} & \bar{V}_{\mathrm{ab}} \\
\bar{V}_{\mathrm{db}} & \bar{V}_{\mathrm{ab}} & \bar{\varepsilon}_{b}
\end{array}\right),
$$

where the diagonal elements $\bar{\varepsilon}_{d}, \bar{\varepsilon}_{a}$, and $\bar{\varepsilon}_{b}$ are energies and the off-diagonal elements $\bar{V}_{\mathrm{da}}, \bar{V}_{\mathrm{db}}$, and $\bar{V}_{\mathrm{ab}}$ are electronic couplings of these diabatic states. Alternatively, applying the two-state model to $\psi_{1}$ and $\psi_{2}$, one obtains diabatic states $\varphi_{d}$ and $\varphi_{a}$ with energies $\varepsilon_{d}$ and $\varepsilon_{a}$ and a coupling matrix element $V_{\text {da }}$,

$$
\left(\begin{array}{ccc}
\varepsilon_{d} & V_{\mathrm{da}} & 0 \\
V_{\mathrm{da}} & \varepsilon_{a} & 0 \\
0 & 0 & \varepsilon_{b}
\end{array}\right) .
$$

In this case, the bridge state $\varphi_{b}$ is identical to the adiabatic state $\psi_{3}$ and $\varepsilon_{b}=E_{3}$. Electronic couplings $V_{\mathrm{da}}$ and $V_{\mathrm{ab}}$ are zero because by definition $\left\langle\psi_{1}|H| \psi_{3}\right\rangle=\left\langle\psi_{2}|H| \psi_{3}\right\rangle=0$ and $\varphi_{d}$ and $\varphi_{a}$ are linear combinations of $\psi_{1}$ and $\psi_{2}$. Let us assume that the states $\varphi_{d}, \varphi_{a}$, and $\varphi_{b}=\psi_{3}$ are zeroth-order approximations to the diabatic states $\bar{\varphi}_{d}, \bar{\varphi}_{a}$, and $\bar{\varphi}_{b}$. We consider a perturbed system where the donor and acceptor states are coupled with the bridge; the matrix elements $V_{\mathrm{db}}^{\prime}$ and $V_{\mathrm{ab}}^{\prime}$ define the coupling. Then, the first-order states of donor and acceptor are

$$
\begin{aligned}
& \tilde{\varphi}_{d} \approx \varphi_{d}+\frac{V_{\mathrm{db}}^{\prime}}{\varepsilon_{d}-\varepsilon_{b}} \varphi_{b}=\varphi_{d}+\frac{V_{\mathrm{db}}^{\prime}}{\Delta_{\mathrm{db}}} \varphi_{b}, \\
& \tilde{\varphi}_{a} \approx \varphi_{a}+\frac{V_{\mathrm{ab}}^{\prime}}{\varepsilon_{a}-\varepsilon_{b}} \varphi_{b}=\varphi_{a}+\frac{V_{\mathrm{ab}}^{\prime}}{\Delta_{\mathrm{ab}}} \varphi_{b},
\end{aligned}
$$

where $\Delta_{\mathrm{db}}$ and $\Delta_{\mathrm{ab}}$ are donor-bridge and acceptor-bridge energy gaps. The electronic coupling $\tilde{V}_{\text {da }}$ between the nonorthogonal states $\tilde{\varphi}_{d}$ and $\tilde{\varphi}_{a}$ is given by ${ }^{5}$

$$
\tilde{V}_{\mathrm{da}}=\left\langle\widetilde{\varphi}_{d} H \widetilde{\varphi}_{a}\right\rangle-1 / 2\left\langle\widetilde{\varphi}_{d} \widetilde{\varphi}_{a}\right\rangle\left(\left\langle\widetilde{\varphi}_{d} H \widetilde{\varphi}_{d}\right\rangle+\left\langle\widetilde{\varphi}_{a} H \widetilde{\varphi}_{a}\right\rangle\right) .
$$

Substituting Eq. (2) for $\widetilde{\varphi}_{d}$ and $\widetilde{\varphi}_{a}$ in Eq. (3) and keeping only terms of first and second order, we obtain

$$
\begin{aligned}
& \left\langle\widetilde{\varphi}_{d} H \widetilde{\varphi}_{a}\right\rangle=V_{\mathrm{db}}+V_{\mathrm{db}}^{\prime} V_{\mathrm{ab}}^{\prime}\left(\frac{1}{\Delta_{\mathrm{db}}}+\frac{1}{\Delta_{\mathrm{ab}}}+\frac{\varepsilon_{b}}{\Delta_{\mathrm{db}} \Delta_{\mathrm{ab}}}\right), \\
& \left\langle\widetilde{\varphi}_{d} \widetilde{\varphi}_{a}\right\rangle\left(\left\langle\widetilde{\varphi}_{d} H \widetilde{\varphi}_{d}\right\rangle+\left\langle\widetilde{\varphi}_{a} H \widetilde{\varphi}_{a}\right\rangle\right)=\frac{V_{\mathrm{db}}^{\prime} V_{\mathrm{ab}}^{\prime}}{\Delta_{\mathrm{db}} \Delta_{\mathrm{ab}}}\left(\varepsilon_{d}+\varepsilon_{a}\right) .
\end{aligned}
$$

Then, combining Eqs. (4) and (5),

$$
\tilde{V}_{\mathrm{da}}=V_{\mathrm{da}}+V_{\mathrm{db}}^{\prime} V_{\mathrm{ab}}^{\prime}\left(\frac{1}{2 \Delta_{\mathrm{db}}}+\frac{1}{2 \Delta_{\mathrm{ab}}}\right) \text {. }
$$

Since

$$
\begin{aligned}
\left(\frac{1}{2 \Delta_{\mathrm{db}}}+\frac{1}{2 \Delta_{\mathrm{ab}}}\right) & =\frac{\varepsilon_{d}+\varepsilon_{a}-2 \varepsilon_{b}}{2\left(\varepsilon_{d}-\varepsilon_{b}\right)\left(\varepsilon_{a}-\varepsilon_{b}\right)} \\
& \approx\left(\frac{\varepsilon_{d}+\varepsilon_{a}}{2}-\varepsilon_{b}\right)^{-1},
\end{aligned}
$$

$E_{3}=\varepsilon_{b}$, and $E_{1}+E_{2}=\varepsilon_{d}+\varepsilon_{a}$ (the trace of a matrix is invariant under a unitary transformation), we obtain

$$
\tilde{V}_{\mathrm{da}} \approx V_{\mathrm{da}}+V_{\mathrm{db}}^{\prime} V_{\mathrm{ab}}^{\prime}\left(\frac{E_{1}+E_{2}}{2}-E_{3}\right)^{-1}=V_{\mathrm{da}}+\frac{V_{\mathrm{db}}^{\prime} V_{\mathrm{ab}}^{\prime}}{E_{\mathrm{gap}}},
$$

where 


$$
E_{\text {gap }}=E_{3}-\frac{E_{1}+E_{2}}{2} .
$$

Thus, the effect of a third state on the donor-acceptor coupling can be approximately described by a superexchange correction. It should be noted that both three- and two-state models treat the same adiabatic states $\psi_{1}, \psi_{2}$, and $\psi_{3}$ of the whole $d-b-a$ system, where all interactions between donor, bridge, and acceptor subunits are accounted for. If the "direct" coupling of donor and acceptor $V_{\mathrm{da}}$ dominate the superexchange term, the two-state model will provide reliable results. However, when the two contributions in $\widetilde{V}_{\mathrm{da}}$ are of the same magnitude, their interference can considerably affect the coupling. Especially strong effects are expected when the terms interfere destructively. In this case $\widetilde{V}_{\mathrm{da}}$ will be much smaller than the two-state coupling $V_{\mathrm{da}}$. Thus, admixture of a bridge state to adiabatic states $\psi_{1}$ and $\psi_{2}$ can considerably restrict the application of the two-state approach. Numerical results presented in a subsequent section show that in most cases when the two-state approach fails to provide reasonable estimates, the superexchange correction of the two-state scheme [Eq. (7)] allows one to obtain accurate values of the donor-acceptor coupling.

A detailed formulation of effective two-state models based on perturbation theory was presented by Newton. ${ }^{5} \mathrm{Al}-$ ternative routes which result to a similar expression for the superexchange term were considered. General expressions for electronic coupling in donor-bridge-acceptor systems were derived using different techniques. ${ }^{5,16-18}$ There is a remarkable difference between the current analysis and the previous results. Usually considering superexchange interaction one operates with the diabatic state of isolated subunits. In such cases a tight-binding approximation is used. In particular, the direct coupling of donor and acceptor is neglected because bridge-mediated superexchange coupling clearly dominates this term. On the contrary, a two-state model, which is based on adiabatic states of donor and acceptor, implies that all bridge effects are implicitly included. However, as noted by Cave and Newton, ${ }^{9}$ multistate effects may essentially influence two-state results. Recently, the systematic study of DNA $\pi$ stacks $^{14}$ has demonstrated that in many cases the two-state model fails to provide accurate estimates of electronic couplings and a multistate approach must be employed for an explicit treatment of bridge states. As we discussed above, the bridge effects may be accounted for in terms of the superexchange model [see Eq. (7)].

\section{Formula for the donor-acceptor coupling}

So far, we did not specify how to determine the matrix elements $V_{\mathrm{db}}^{\prime}$ and $V_{\mathrm{ab}}^{\prime}$. The GMH method offers a convenient way to estimate these quantities. Because for ground-state thermal processes the diabatic and adiabatic states essentially coincide at equilibrium configurations, ${ }^{5}$ applying the twostate GMH model [Eq. (1)] to pairs of states $\left(\psi_{1}, \psi_{2}\right)$, $\left(\psi_{1}, \psi_{3}\right)$ and $\left(\psi_{2}, \psi_{3}\right)$ may express the matrix elements $V_{\mathrm{da}}$, $V_{\mathrm{db}}^{\prime}$, and $V_{\mathrm{ab}}^{\prime}$ involved in Eq. (7). Then

$$
\tilde{V}_{\mathrm{da}}=\frac{\left(E_{2}-E_{1}\right) \mu_{12}}{\left(\mu_{a}-\mu_{b}\right)}+\frac{\left(E_{3}-E_{1}\right) \mu_{13}}{\left(\mu_{b}-\mu_{d}\right)} \frac{\left(E_{3}-E_{2}\right) \mu_{23}}{\left(\mu_{a}-\mu_{b}\right)} \frac{1}{E_{\text {gap }}} .
$$

Taking into account that

$$
\left(E_{3}-E_{1}\right)\left(E_{3}-E_{2}\right) \approx\left(E_{3}-\frac{E_{2}+E_{2}}{2}\right)^{2}=E_{\text {gap }}^{2}
$$

we obtain

$$
\tilde{V}_{\mathrm{da}}=\left(E_{2}-E_{1}\right) \frac{\mu_{12}}{\mu_{a}-\mu_{d}}+E_{\mathrm{gap}} \frac{\mu_{13}}{\mu_{b}-\mu_{d}} \frac{\mu_{23}}{\mu_{a}-\mu_{b}} .
$$

The differences of diabatic dipole moments can be expressed via adiabatic matrix elements [see Eq. (1)] or estimated using geometric parameters of the system. While in some cases the adiabatic dipole moment difference can be determined by the Stark spectroscopy, usually these experimental data are not available and estimates based on structural parameters are employed. The diabatic dipole moment difference is set to $e R_{\mathrm{da}}$, where $R_{\mathrm{da}}$ is the distance between donor and acceptor. In general, $R_{\mathrm{da}}$ is not well defined, and therefore the value of $\left(\mu_{a}-\mu_{d}\right)$ is rather inaccurate. However, in $\pi$ stacks, the situation is more favorable. One may employ the projections of adiabatic dipole and transition moments onto an axis perpendicular to the planes of donor and acceptor.

Then, $\mu_{b}-\mu_{d} \approx \mu_{a}-\mu_{b} \approx 1 / 2\left(\mu_{a}-\mu_{d}\right)$ and $\left(\mu_{a}-\mu_{d}\right)$ $=e R_{\mathrm{da}}$, and finally, we have

$$
\tilde{V}_{\mathrm{da}}=\frac{\left(E_{2}-E_{1}\right) \mu_{12}}{R_{\mathrm{da}}}+\frac{4 E_{\mathrm{gap}} \mu_{13} \mu_{23}}{R_{\mathrm{da}}^{2}} .
$$

In this equation, the transition dipole moments and $R_{\mathrm{da}}$ are in atomic units. If the transition moments are in debye and the distance is in angstrom, $R_{\mathrm{da}}$ in Eq. (10) must be replaced by $\left(f R_{\mathrm{da}}\right)$ with $f=4.80324$. Because the distance between the planes of donor and acceptor in $\pi$ stacks is usually well known, expression (10) may be used for estimating couplings on the basis of spectroscopic data.

Expression (9) can be extended to systems containing several bridge units:

$$
\begin{aligned}
\tilde{V}_{\mathrm{da}} \approx & \left(E_{2}-E_{1}\right) \frac{\mu_{12}}{\mu_{a}-\mu_{d}} \\
& +\sum_{j=3}^{n}\left(E_{j}-\frac{E_{1}+E_{2}}{2}\right) \frac{\mu_{1 j}}{\mu_{j}-\mu_{d}} \frac{\mu_{2 j}}{\mu_{a}-\mu_{j}},
\end{aligned}
$$

where the energy $E_{j}$ corresponds to adiabatic state $j$ localized on the bridge. The differences $\mu_{j}-\mu_{d}$ and $\mu_{a}-\mu_{j}$ can be expressed in terms of adiabatic dipole and transition moments [see Eq. (1)].

\section{Diagnostic to determine whether a two-state model is applicable}

As seen from Eq. (9), a two-state model may be applied if the superexchange term is relatively small, i.e.,

$$
\left|\frac{\left(E_{2}-E_{1}\right) \mu_{12}}{\mu_{a}-\mu_{d}}\right| \gg\left|\frac{E_{\mathrm{gap}} E_{13} \mu_{23}}{\left(\mu_{a}-\mu_{b}\right)\left(\mu_{b}-\mu_{d}\right)}\right| .
$$

In $\pi$ stacks, $\mu_{b}-\mu_{d} \approx \mu_{a}-\mu_{b} \approx 1 / 2\left(\mu_{a}-\mu_{d}\right)$, and therefore 


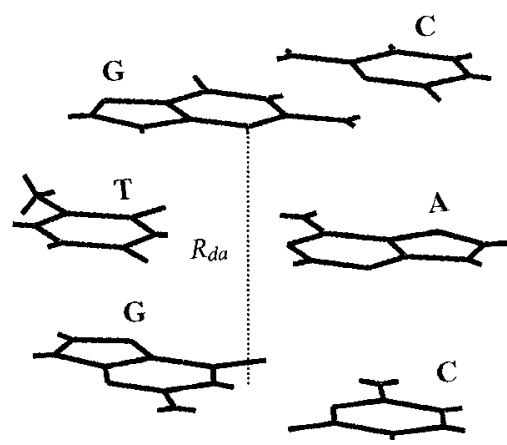

FIG. 1. $\pi$ stack of three Watson-Crick pairs (GC, TA, and GC). The donor and acceptor sites, GC pairs, are separated by a TA bridge. The distances between donor, bridge, and acceptor are defined as spacing between their planes.

$$
\left|\left(E_{2}-E_{1}\right) \mu_{12}\right| \gg\left|\frac{4 E_{\mathrm{gap}} \mu_{13} \mu_{23}}{\left(\mu_{a}-\mu_{d}\right)}\right|
$$

Introducing a parameter $\gamma$

$$
\gamma=1+\frac{\left(E_{2}-E_{1}\right)\left(\mu_{a}-\mu_{d}\right) \mu_{12}}{4 E_{\mathrm{gap}} \mu_{13} \mu_{23}} \approx 1+\frac{\left(E_{2}-E_{1}\right) R_{\mathrm{da}} \mu_{12}}{4 E_{\mathrm{gap}} \mu_{13} \mu_{23}}
$$

condition (11) can be rewritten as $|\gamma| \gg 0$.

When both terms in Eq. (9) are of the same sign, i.e., they interfere constructively, the two-state model will underestimate the donor-acceptor coupling. When $\gamma=1$, the twostate term is zero and the donor-acceptor coupling is controlled by the superexchange term; $\gamma<1$ indicates that the direct and superexchange terms interfere destructively (they are of opposite signs) and the two-state approach will overestimate the coupling. The two-state model becomes completely unreliable when the terms are similar in magnitude but opposite in sign, $\gamma \approx 0$. In this case, the multistate GMH scheme must be employed.

\section{Effects of external parameters on adiabatic and diabatic properties}

In this section we show that small fluctuations of external parameters may cause very big changes in adiabatic dipole matrix. Let us consider the effect of the external field on the adiabatic and diabatic quantities of a stack consisting of three nucleobase pairs. Two guanine-cytosine (GC) pairs are the donor and acceptor sites separated by a thymine-adenine (TA) bridge. The structure of the stack is shown in Fig. 1. The distance between base pairs in the stack is set to $3.38 \AA$. To this end we carried out quantum-chemical calculations using the Hartree-Fock method with the standard $6-31 G^{*}$ basis set as described in previous papers. ${ }^{14,19}$ A weak electric field along the stack axis will be used as the external parameter. The three-state GMH method was employed to estimate the coupling matrix elements. The results of calculations are given in Table I. As can be seen, the electric field considerably affects the charge distribution between donor and acceptor and adiabatic dipole matrix elements.

When the electric field is zero, $F=0$, donor and acceptor are off resonance; the energy mismatch $\varepsilon_{a}-\varepsilon_{d}$ is $20.4 \mathrm{meV}$. In the ground state, the excess charge is almost completely localized on the donor. The charges on the acceptor and bridge sites are found to be 0.007 and 0.010 , respectively. Electron transition $\psi_{1} \rightarrow \psi_{2}$ results in a considerable change of the adiabatic dipole moment $\mu_{2}-\mu_{1} \approx 31.8 \mathrm{D}$. Because the state $\psi_{3}$ is mainly localized on the bridge, $\mu_{3}-\mu_{1}=\mu_{2}$ $-\mu_{3}=1 / 2\left(\mu_{2}-\mu_{1}\right)$. The transition moment $\mu_{12}$ of $\sim 2.7 \mathrm{D}$ is essentially larger than $\mu_{13}$ and $\mu_{23}$. The $d$ - $a$ electronic coupling $\bar{V}_{\mathrm{da}}$ is by order of magnitude smaller than the matrix elements $\bar{V}_{\mathrm{db}}$ and $\bar{V}_{\mathrm{ab}}$ associated with the bridge state.

By applying an electric field of $6.1 \times 10^{-5}$ a.u. the energy mismatch between $d$ and $a$ levels essentially decreases, $\varepsilon_{a}$ $-\varepsilon_{d}=2 \mathrm{meV}$. As can be seen from Table I, the adiabatic dipole and transition moments are very responsive to the external perturbation. However, the electronic couplings in the system retain their values. When the electric field is 6.725 $\times 10^{-5}$ a.u., the donor and acceptor states are almost in reso-

TABLE I. Dependence of adiabatic and diabatic parameters of the GTG stack on external electric field (in $10^{-5}$ a.u.).

\begin{tabular}{lccccc}
\hline \hline Parameter & $F=0$ & $F=6.100$ & $F=6.725$ & $F=7.000$ & $F=13.450$ \\
\hline$E_{2}-E_{1}(\mathrm{meV})$ & 20.7 & 3.50 & 3.27 & 3.27 & 20.4 \\
$q_{d}$ & 0.984 & 0.747 & 0.499 & 0.375 & 0.006 \\
$q_{a}$ & 0.007 & 0.243 & 0.491 & 0.615 & 0.987 \\
$q_{b}$ & 0.010 & 0.010 & 0.010 & 0.010 & 0.007 \\
$\mu_{2}-\mu_{1}(\mathrm{D})$ & 31.77 & 16.43 & 0.29 & -7.76 & -31.83 \\
$\mu_{3}-\mu_{1}(\mathrm{D})$ & 15.89 & 8.22 & 0.14 & -3.88 & -15.91 \\
$\mu_{3}-\mu_{2}(\mathrm{D})$ & -15.89 & -8.22 & -0.14 & 3.88 & 15.91 \\
$\mu_{12}(\mathrm{D})$ & 2.68 & 13.86 & 16.11 & -15.64 & -2.54 \\
$\mu_{13}(\mathrm{D})$ & 0.51 & 0.67 & 0.71 & 0.71 & 0.57 \\
$\mu_{23}(\mathrm{D})$ & 0.51 & 0.24 & 0.05 & 0.04 & 0.43 \\
$\mu_{a}-\mu_{d}(\mathrm{D})$ & 32.23 & 32.23 & 32.23 & 32.23 & 32.23 \\
$\varepsilon_{a}-\varepsilon_{d}(\mathrm{meV})$ & 20.4 & 2.0 & 0.2 & -0.6 & -20.0 \\
$\bar{V}_{\mathrm{da}}(\mathrm{meV})$ & 2.46 & 2.26 & 2.37 & 2.32 & 2.34 \\
$\bar{V}_{\mathrm{db}}(\mathrm{meV})$ & 22.0 & 22.0 & 22.0 & 22.0 & 22.0 \\
$\bar{V}_{\mathrm{ab}}(\mathrm{meV})$ & 25.4 & 25.4 & 25.4 & 25.4 & 25.4 \\
\hline
\end{tabular}


TABLE II. Comparison of donor-acceptor electronic couplings in $d$ - $b$ - $a$ stacks with $E_{2}-E_{1}=0.1 \mathrm{eV}$ and $E_{\text {gap }}$ $=1.0 \mathrm{eV}$. The couplings $V_{\mathrm{da}}, \bar{V}_{\mathrm{da}}$, and $\widetilde{V}_{\mathrm{da}}$ are calculated by using the two- and three-state GMH schemes and Eq. (9), respectively.

\begin{tabular}{cccccccc}
\hline \hline \multirow{2}{*}{ System } & $\mu_{12}(\mathrm{D})$ & $\mu_{13}(\mathrm{D})$ & $\mu_{23}(\mathrm{D})$ & $\left|\bar{V}_{\mathrm{da}}\right|(\mathrm{meV})$ & $|\gamma|$ & $\left|V_{\mathrm{da}} / \bar{V}_{\mathrm{da}}\right|$ & $\left|\widetilde{V}_{\mathrm{da}} / \bar{V}_{\mathrm{da}}\right|$ \\
\hline 1 & 5.0 & 1.0 & 1.0 & 18.80 & 5.08 & 0.82 & 1.01 \\
2 & 5.0 & 1.0 & -1.0 & 11.71 & 3.30 & 1.31 & 0.99 \\
3 & 2.5 & 1.0 & 1.0 & 11.30 & 3.04 & 0.68 & 1.01 \\
4 & 2.5 & 1.0 & -1.0 & 3.95 & 1.04 & 1.94 & 0.99 \\
5 & 1.3 & 1.0 & 1.0 & 7.68 & 2.06 & 0.52 & 1.01 \\
6 & 1.3 & 1.0 & -1.0 & 0.26 & 0.06 & 15.60 & 0.90 \\
7 & 1.0 & 1.0 & 1.0 & 6.77 & 1.82 & 0.453 & 1.01 \\
8 & 1.0 & 1.0 & -1.0 & 0.66 & 0.18 & 4.61 & 1.04 \\
9 & 0.5 & 1.0 & 1.0 & 5.25 & 1.41 & 0.29 & 1.01 \\
10 & 0.5 & 1.0 & -1.0 & 2.20 & 0.59 & 0.70 & 1.01 \\
11 & 1.0 & 0.1 & 1.0 & 3.32 & 9.16 & 0.92 & 1.04 \\
12 & 1.0 & 0.1 & -1.0 & 2.57 & 7.16 & 1.19 & 1.05 \\
13 & 0.0 & 1.0 & \pm 1.0 & 3.06 & 0.0 & 0.0 & 1.01 \\
14 & 1.0 & 0.0 & 1.0 & 2.94 & Inf & 1.04 & 1.04 \\
15 & 1.0 & 0.0 & 0.0 & 3.06 & Inf & 1.00 & 1.00 \\
\hline \hline
\end{tabular}

nance. The diabatic energy gap $\varepsilon_{a}-\varepsilon_{d}$ is calculated to be $0.2 \mathrm{meV}$; the positive charge is equally distributed between donor and acceptor, and the dipole moment difference $\mu_{2}$ $-\mu_{1}$ decreases to $0.29 \mathrm{D}$. On the contrary, the transition moment $\mu_{12}$ becomes very large, $\sim 16 \mathrm{D}$. By further increase of the electric field, the acceptor state $d-b-a^{+}$becomes lower in energy than the donor state $d^{+}-b-a$ and corresponds now to the ground state. The difference of adiabatic dipole moment changes its sign. The properties calculated at $F=13.450$ $\times 10^{-5}$ a.u. are similar to those obtained at $F=0$, provided that the donor and acceptor states are exchanged.

As seen from Table I, the properties of the diabatic states remain almost unchanged despite the fact that adiabatic dipole matrix elements are very sensitive to the external perturbation. The calculated differences of diabatic dipole moments $\mu_{a}-\mu_{d}=32.23 \mathrm{D}$ and $\mu_{b}-\mu_{d}=\mu_{a}-\mu_{b}=16.11 \mathrm{D}$ are practically independent of the applied electric field. The calculated value $\mu_{a}-\mu_{d}=32.23 \mathrm{D}$ is in very good agreement with an estimate $e R_{\mathrm{da}}=32.47 \mathrm{D}\left(R_{\mathrm{da}}=6.68 \AA\right)$. The couplings $\bar{V}_{\mathrm{db}}$ and $\bar{V}_{\mathrm{ab}}$ also retain their values. Although $\bar{V}_{\mathrm{da}}$ is found to be more sensitive than $\bar{V}_{\mathrm{db}}$ and $\bar{V}_{\mathrm{ab}}$, its variation is rather insignificant.

Overall, the results demonstrate that small fluctuations of donor and acceptor energy mismatch due to external perturbations may cause considerable changes in the adiabatic dipole and transition moments, while electronic couplings remain almost unchanged. Therefore, varying an external parameter (electric field, geometry of donor or acceptor, and position of solvent molecules), one can essentially change adiabatic dipole and transition moments. Because of that, a ratio of these quantities cannot be used, in general, to predict the role of the bridge effects. Lambert et al. concluded that the two-state model is a good approximation only when the difference of dipole moments of donor and acceptor is large compared to transition moments associated with a bridge state. $^{15}$ This is the case when $F=0$ (Table I). The donoracceptor couplings calculated using the two- and three-state schemes are 1.72 and $2.46 \mathrm{meV}$, respectively. If donor and acceptor are almost in resonance $\left(F=6.725 \times 10^{-5}\right.$ a.u. $)$, the difference $\mu_{2}-\mu_{1}=0.29 \mathrm{D}$ is remarkably smaller than $\mu_{13}$ $=0.71 \mathrm{D}$. However, the two- and three-state couplings are similar $(1.63$ and $2.37 \mathrm{meV})$ to the estimates found at $F=0$. Furthermore, according to our experience, in nonsymmetric systems the difference $\mu_{2}-\mu_{1}$ is often essentially larger than transition moments; however, the performance of the twostate model strongly depends on the system being considered.

\section{Numerical estimation}

As shown above, adiabatic dipole matrix elements in a $d-b-a$ system can vary in a wide range depending on external parameters, while electronic couplings essentially retain their magnitude. Moreover, structural fluctuations in $\pi$ stacks are found to cause considerable changes in the electronic coupling. ${ }^{20,21}$ Thus, adiabatic transition dipole moments appear to be very sensitive to structural features of $\pi$ stacks. Now we compare electronic couplings $\widetilde{V}_{\mathrm{da}}, V_{\mathrm{da}}^{\prime}$, and $\bar{V}_{\mathrm{da}}$ estimated by using Eq. (8) and the two- and three-state GMH schemes, respectively. Table II provides numerical results for the couplings calculated for several sets of adiabatic parameters. In the calculations, the difference of diabatic dipole moments $\mu_{a}-\mu_{d}$ was assumed to be $32.66 \mathrm{D}$. In line with calculation results (Table I) we assumed that $\mu_{3}-\mu_{1}=\mu_{2}$ $-\mu_{3}=1 / 2\left(\mu_{2}-\mu_{1}\right)$. In turn, $\left(\mu_{2}-\mu_{1}\right)$ can be estimated as $\mu_{2}-\mu_{1}=\sqrt{\left(\mu_{a}-\mu_{d}\right)^{2}-4 \mu_{12}^{2}}$. These physically reasonable constraints allow us to decrease a number of variable parameters of the model. For all systems we assumed that $E_{2}-E_{1}$ and $E_{\text {gap }}$ are 0.1 and $1.0 \mathrm{eV}$, respectively. Since electronic coupling depends on the product $\left(E_{j}-E_{i}\right) \mu_{i j}$, similar values of the coupling can be found in systems with quite different adiabatic properties when the corresponding products are close to each other.

In system 1, all transition dipole moments are positive, and consequently, both terms in Eq. (9) interfere constructively. The electronic coupling $\bar{V}_{\mathrm{da}}$ calculated with the three- 
state approach is $18 \mathrm{meV}$. As can be seen from Table II, the two-state scheme underestimates the matrix element by about $20 \%$. The value $\widetilde{V}_{\text {da }}$ given by Eq. (10) deviates from $\bar{V}_{\text {da }}$ by less than $1 \%$. In system $2, \mu_{13}$ is negative and the coupling becomes smaller when the superexchange correction is accounted for, $V_{\mathrm{da}}>\widetilde{V}_{\mathrm{da}} \approx \bar{V}_{\mathrm{da}}$. In this case the twostate model overestimates the coupling by $30 \%$. Less accurate values are provided by the two-state approach for systems 3 and 4 with $\mu_{12}=2.5 \mathrm{D}$-the coupling is underestimated by $30 \%$ in system 3 and predicted to be almost twice as large in system 4. In both cases, Eq. (9) gives accurate estimates of the electronic coupling. When $\mu_{12}=1.3 \mathrm{D}$ (systems 5 and 6 in Table II), the absolute values of $\bar{V}_{\mathrm{da}}$ and the correction term are very similar. Because of that, in system 5 $V_{\mathrm{da}}$ is found to be equal only to half of the reference value $\bar{V}_{\text {da }}$. In system $6, \gamma \approx 0$ and the coupling matrix element is considerably (by a factor of 15) overestimated if the twostate GMH model is employed. By contrast, the $\tilde{V}_{\mathrm{da}}$ and $\bar{V}_{\mathrm{da}}$ values are in good agreement. While the two-state approach shows better performance for systems 7-10 the estimates of the coupling are not very accurate. Note that in all these cases, Eq. (9) provides reliable results. In systems 11 and 12, $\mu_{13}$ is an order of magnitude smaller than $\mu_{12}$ and $\mu_{23}$. Because the parameter $\gamma$ in these cases is relatively large, $|\gamma|$ $>7$, the superexchange term in Eq. (9) is essentially smaller than the direct coupling, and the two-state model gives good estimates.

Finally, we consider three special cases. In system 13 with $\mu_{12}=0$, the parameter $\gamma$ is zero. It means that the $d$ - $a$ coupling is exclusively defined by superexchange correction. Obviously, the two-state scheme cannot be applied in this case. By contrast, in system 14, the superexchange term in Eq. (9) is zero since $\mu_{13}=0$, the parameter $\gamma$ is infinitely large, and the two-state model provides a good estimate for the coupling, $V_{\mathrm{da}} \approx \bar{V}_{\mathrm{da}}$. While $\mu_{13}=0$, the donor-acceptor coupling $\bar{V}_{\mathrm{da}}$ calculated within the three-state approach depends on $\mu_{23}$. However, this effect will not be reproduced by formula (9). System 15 with $\mu_{13}=\mu_{23}=0$ represents a rather unlikely situation. In terms of the GMH scheme, the bridge state is completely uncoupled and the three-state model reduces to a two-state model. It is apparent that in this case the two-state approach provides accurate values of the donoracceptor coupling $V_{\mathrm{da}}=\bar{V}_{\mathrm{da}}$.

Overall, based on the numerical results presented in Table II, we conclude that estimation of the donor-acceptor coupling can be considerably improved by employing Eq. (9) instead of the two-state model. However, when $\gamma \approx 0$, the formula may provide values which are not very accurate, and therefore the three-state GMH treatment has to be applied in such cases.

\section{CONCLUSIONS}

We analyzed the difference in donor and acceptor electronic couplings calculated within two- and three-state schemes based on the generalized Mulliken-Hush approach. It has been shown that admixture of a bridge state to adiabatic states of donor and acceptor considerably affects the coupling matrix element derived within the two-state model, and thus the two-state treatment is limited in use. Using perturbation theory, we found that the effects of the bridge states can be described in terms of superexchange interaction [Eq. (9)]. Based on numerical results we showed that the superexchange correction considerably improves estimates of the donor-acceptor coupling derived within a two-state approach. In most cases where the two-state scheme fails, Eq. (9) gives reliable values which are in good agreement (within $5 \%$ ) with the results of the three-state GMH model.

In $\pi$-stacked $d-b$ - $a$ systems, an excess charge migrates along the axis perpendicular to the planes of donor and acceptor. In this special case, the difference of diabatic dipole moments is well defined by the spacing between the planes, and therefore Eq. (12) may be employed.

We demonstrated that small variations of an external parameter, which affects the splitting of donor and acceptor levels, may considerably change charge distribution and adiabatic dipole matrix elements, while electronic couplings remain almost unchanged. Because of that, the question whether a two-state GMH model is applicable cannot be answered when only adiabatic dipole and transition moments are compared. A diagnostic, where the parameter $\gamma$ is defined by Eq. (14), has been proposed to determine whether the two-state model is a good approximation. It is suggested that the two-state treatment provides reliable results when $|\gamma|$ $>5$; however, it becomes rather unusable if $|\gamma|<2$.

\section{ACKNOWLEDGMENT}

This work has been supported by the Spanish Ministerio de Educación y Ciencia (Project No. CTQ2005-04563).

${ }^{1}$ V. Lemaur, M. Steel, D. Beljonne, J. L. Bredas, and J. Cornil, J. Am. Chem. Soc. 127, 6077 (2005).

${ }^{2}$ Long-Range Charge Transfer in DNA: Topics in Current Chemistry, edited by G. B. Shuster (Springer, Berlin, 2004), Vols. 236 and 237.

${ }^{3}$ M. K. Lee, M. J. Shephard, S. M. Risser, S. Priyadarshy, M. N. PaddonRow, and D. N. Beratan, J. Phys. Chem. A 104, 7593 (2000).

${ }^{4}$ Y. A. Berlin, I. V. Kurnikov, D. N. Beratan, M. A. Ratner, and A. L. Burin, in Long-Range Charge Transfer in DNA: Topics in Current Chemistry, edited by G. B. Shuster (Springer, Berlin, 2004), Vol. 237, pp. 1-36.

${ }^{5}$ M. D. Newton, Chem. Rev. (Washington, D.C.) 91, 767 (1991).

${ }^{6}$ M. D. Newton, Adv. Chem. Phys. 106, 303 (1999).

${ }^{7}$ N. Rösch and A. A. Voityuk, in Long-Range Charge Transfer in DNA: Topics in Current Chemistry, edited by G. B. Shuster (Springer, Berlin, 2004), Vol. 237, pp. 37-72.

${ }^{8}$ R. J. Cave and M. D. Newton, Chem. Phys. Lett. 249, 15 (1996).

${ }^{9}$ R. J. Cave and M. D. Newton, J. Chem. Phys. 106, 9213 (1997).

${ }^{10}$ D. Beljonne, G. Pourtois, M. A. Ratner, and J. L. Bredas, J. Am. Chem. Soc. 125, 14510 (2003).

${ }^{11}$ J. R. Zheng, Y. K. Kang, M. J. Therien, and D. N. Beratan, J. Am. Chem. Soc. 127, 11303 (2005).

${ }^{12}$ A. A. Voityuk, J. Chem. Phys. 123, 034903 (2005).

${ }^{13}$ M. Rust, J. Lappe, and R. J. Cave, J. Phys. Chem. A 106, 3930 (2002).

${ }^{14}$ A. A. Voityuk, J. Phys. Chem. B 109, 17917 (2005).

${ }^{15}$ C. Lambert, S. Amthor, and J. Schelter, J. Phys. Chem. A 108, 6474 (2004).

${ }^{16}$ S. Larsson, J. Am. Chem. Soc. 103, 4034 (1981).

${ }^{17}$ M. A. Ratner, J. Phys. Chem. 94, 4877 (1990).

${ }^{18}$ S. S. Skourtis, D. N. Beratan, and J. N. Onuchic, Chem. Phys. 176, 501 (1993).

${ }^{19}$ A. A. Voityuk, J. Jortner, M. Bixon, and N. Rösch, J. Chem. Phys. 114, $5614(2001)$.

${ }^{20}$ A. Troisi and G. Orlandi, J. Phys. Chem. B 106, 2093 (2002).

${ }^{21}$ A. A. Voityuk, K. Siriwong, and N. Rosch, Phys. Chem. Chem. Phys. 3, $5421(2001)$ 